\title{
Video Article \\ Characterization of Proteins by Size-Exclusion Chromatography Coupled to Multi-Angle Light Scattering (SEC-MALS)
}

\author{
Daniel Some ${ }^{1}$, Hadar Amartely ${ }^{2}$, Ayala Tsadok $^{3}$, Mario Lebendiker $^{2}$ \\ ${ }^{1}$ Wyatt Technology Corporation \\ ${ }^{2}$ Wolfson Centre for Applied Structural Biology, The Alexander Silberman Institute of Life Science, The Hebrew University of Jerusalem \\ ${ }^{3}$ Danyel Biotech Ltd.
}

Correspondence to: Daniel Some at dsome@wyatt.com

URL: https://www.jove.com/video/59615

DOI: doi:10.3791/59615

Keywords: Biochemistry, Issue 148, size-exclusion chromatography, multi-angle light scattering (MALS), protein characterization, molecular weight, bovine serum albumin, oligomers, aggregates, protein-protein complexes, quality control

Date Published: 6/20/2019

Citation: Some, D., Amartely, H., Tsadok, A., Lebendiker, M. Characterization of Proteins by Size-Exclusion Chromatography Coupled to Multi-Angle Light Scattering (SEC-MALS). J. Vis. Exp. (148), e59615, doi:10.3791/59615 (2019).

\section{Abstract}

Analytical size-exclusion chromatography (SEC), commonly used for the determination of the molecular weight of proteins and protein-protein complexes in solution, is a relative technique that relies on the elution volume of the analyte to estimate molecular weight. When the protein is not globular or undergoes non-ideal column interactions, the calibration curve based on protein standards is invalid, and the molecular weight determined from elution volume is incorrect. Multi-angle light scattering (MALS) is an absolute technique that determines the molecular weight of an analyte in solution from basic physical equations. The combination of SEC for separation with MALS for analysis constitutes a versatile, reliable means for characterizing solutions of one or more protein species including monomers, native oligomers or aggregates, and heterocomplexes. Since the measurement is performed at each elution volume, SEC-MALS can determine if an eluting peak is homogeneous or heterogeneous and distinguish between a fixed molecular weight distribution versus dynamic equilibrium. Analysis of modified proteins such as glycoproteins or lipoproteins, or conjugates such as detergent-solubilized membrane proteins, is also possible. Hence, SEC-MALS is a critical tool for the protein chemist who must confirm the biophysical properties and solution behavior of molecules produced for biological or biotechnological research. This protocol for SEC-MALS analyzes the molecular weight and size of pure protein monomers and aggregates. The data acquired serve as a foundation for further SEC-MALS analyses including those of complexes, glycoproteins and surfactant-bound membrane proteins.

\section{Video Link}

The video component of this article can be found at https://www.jove.com/video/59615/

\section{Introduction}

Reliable analysis of the molecular weight (MW) of proteins in solution is essential for biomolecular research ${ }^{1,2,3,4}$. MW analysis informs the scientist if the correct protein has been produced and if it is suitable for use in further experimentation ${ }^{5,6}$. As described on the web sites of protein research networks P4EU ${ }^{7}$ and ARBRE-Mobieu ${ }^{8}$, protein quality control must characterize not only the purity of the final product, but also its oligomeric state, homogeneity, identity, conformation, structure, post-translation modifications and other properties.

MW measurement in non-denaturing solution identifies the form of the protein that is present in an aqueous environment, whether monomeric or oligomeric. While for many proteins the goal is to produce the monomeric form, for others a specific native oligomer is key to biological activity $9,10,11,12$. Other oligomers and non-native aggregates are undesirable and will lead to flaws in structural determination by crystallography, nuclear magnetic resonance (NMR) or small-angle X-ray scattering, as well as artifacts or inaccuracies in functional assays to quantify binding by isothermal titration calorimetry or surface plasmon resonance ${ }^{2,13}$.

In the case of biotherapeutics such as monoclonal antibodies (mAbs), solution-based MW analysis serves a similar purpose of quality control and product characterization. Excessive aggregates and fragments are indicative of an unstable product that is not suitable for human use. Regulatory agencies require careful characterization, not only of the therapeutic molecule but also potential degradants that may be present in the final product ${ }^{14,15,16,17}$.

Some of the most widespread methods for analyzing protein MW are sodium dodecyl sulfate polyacrylamide gel electrophoresis (SDS-PAGE), capillary electrophoresis (CE), native PAGE, mass spectrometry (MS), size-exclusion chromatography (SEC) and analytical ultracentrifugation (AUC). Of these, SDS-PAGE, CE and MS are not performed in the native state and typically lead to dissociation of oligomers and aggregates, hence are not suitable for determining the native oligomer or quantifying aggregates. Although native PAGE does, theoretically, retain the native state, in our experience it is difficult to optimize for many proteins, and results are not very reliable. AUC, whether by sedimentation velocity or 
sedimentation equilibrium, is quantitative and can determine MW from first principles, but it is quite cumbersome, requiring much manual labor and significant expertise in data interpretation, long experiment time and a very expensive instrument.

Analytical SEC is a quantitative and relatively robust, simple method that separates macromolecules during flow through a packed column. The principles and applications of SEC are well presented in several reviews ${ }^{18,19,20}$ and in the handbook "Size Exclusion Chromatography: Principles and Methods"21. The differences in retention are due to different amounts of time spent diffusing into and out of the pores in the stationary phase before eluting from the end of the column. The differences arise (nominally) from the relative sizes and diffusion coefficients of the molecules ${ }^{22}$. A calibration curve is constructed using a series of reference molecules, relating the MW of the molecule to elution volume. For proteins, the reference molecules are generally well-behaved, globular proteins that do not interact with the column via charge or hydrophobic surface residues. Elution volume is measured with an ultraviolet (UV) absorbance detector. If the UV extinction coefficient is known-often calculated from the sequence-the protein peak total mass may also be quantitated.

Notably, the analysis of MW by SEC relies on two key assumptions regarding the proteins to be characterized: 1) they share with the reference standards the same conformation and specific volume (in other words, the same relationship between diffusion properties and MW) and 2) like the reference standards, they do not interact with the column except by steric properties-they do not adhere to the column packing by charge or hydrophobic interactions. Deviations from these assumptions invalidate the calibration curve and lead to erroneous MW determinations. This is the case for intrinsically disordered proteins that have large Stokes radii due to their extensive unstructured regions ${ }^{23,24}$ or non-spherical/linear $^{2}$ oligomeric assemblies ${ }^{10}$. Glycosylated proteins will generally have a larger Stokes radius than the non-glycosylated form, even when the added carbohydrate mass is taken into account ${ }^{19}$. Detergent-solubilized membrane proteins elute differently than calibration proteins because their elution from SEC depends on the total size of the polypeptide-detergent-lipids complex rather than the oligomeric state and molar mass of the protein $^{25,26}$. Column chemistry, $\mathrm{pH}$ and salt conditions all affect elution volumes of proteins with charged or hydrophobic surface residues ${ }^{27,28}$.

SEC becomes much more versatile and reliable for MW determination when combined with multi-angle light scattering (MALS) and differential refractive index $(\mathrm{dRI})$ detectors ${ }^{3,4,11,29,30,31,32}$. A dRI detector determines concentration based on the change in solution refractive index due to the presence of the analyte. A MALS detector measures the proportion of light scattered by an analyte into multiple angles relative to the incident laser beam. Collectively known as SEC-MALS, this instrumentation determines MW independently of elution time since MW can be calculated directly from first principles using Equation 1 ,

$$
M=\frac{R(0)}{K c\left(\frac{d n}{d c}\right)^{2}}
$$

where $M$ is the molecular weight of the analyte, $R(0)$ the reduced Rayleigh ratio (i.e., the amount of light scattered by the analyte relative to the laser intensity) determined by the MALS detector and extrapolated to angle zero, $c$ the weight concentration determined by the UV or dRI detector, $d n / d c$ the refractive index increment of the analyte (essentially the difference between the refractive index of the analyte and the buffer), and $K$ an optical constant that depends on the system properties such as wavelength and solvent refractive index ${ }^{29}$.

In SEC-MALS, the SEC column is used solely to separate the various species in solution so that they enter the MALS and concentration detector cells individually. The actual retention time has no significance for the analysis except as far as how well it resolves the protein species. The instruments are calibrated independently of the column and do not rely on reference standards. Hence, SEC-MALS is considered an 'absolute' method for MW determination from basic physical equations. If the sample is heterogeneous and not completely separated by the column, then the value provided at each elution volume will be a weight average of the molecules in each elution volume that flows through the flow cell per time slice, approximately $75 \mu \mathrm{L}$.

By analysis of the angular variation of scattering intensity, MALS can also determine the size (root-mean-square radius, $R_{\mathrm{g}}$ ) of macromolecules and nanoparticles with geometric radius larger than about $12.5 \mathrm{~nm}^{29}$. For smaller species such as monomeric proteins and oligomers, a dynamic light scattering (DLS) module may be added to the MALS instrument in order to measure hydrodynamic radii from $0.5 \mathrm{~nm}$ and up ${ }^{33}$.

While either UV or dRI concentration analysis may provide the value of $c$ in Eq. 1, use of $\mathrm{dRI}$ is preferred for two reasons: 1) dRI is a universal concentration detector, suitable for analyzing molecules such as sugars or polysaccharides that do not contain a UV chromophore 34 ; and 2) the concentration response $d n / d c$ of almost all pure proteins in aqueous buffer is the same to within one or two percent $(0.185 \mathrm{~mL} / \mathrm{g})^{35}$, so there is no need to know the UV extinction coefficient.

The use of SEC-MALS in protein research is quite extensive. By far the most common applications are establishing whether a purified protein is monomeric or oligomeric and the degree of oligomerization, and assessing aggregates ${ }^{3,10,11,17,31,36,37,38}$. The ability to do so for detergentsolubilized membrane proteins that cannot be characterized by traditional means is especially prized, and detailed protocols for this have been published ${ }^{31,39,40,41,42,43}$. Other common applications include establishing the degree of post-translational modification and polydispersity of glycoprotein, lipoproteins and similar conjugates ${ }^{4,31,44,45,46,47}$; the formation (or lack thereof) and absolute stoichiometry (as opposed to stoichiometric ratio) of heterocomplexes including protein-protein, protein-nucleic acid and protein-polysaccharide complexes ${ }^{24,46,48,49,50,51,52 .}$ determining the monomer-dimer equilibrium dissociation constant ${ }^{99,53,54}$; and evaluating protein conformation ${ }^{55,56}$. Beyond proteins, SEC-MALS is invaluable for characterization of peptides ${ }^{57,58}$, broadly heterogeneous natural polymers such as heparins ${ }^{59}$ and chitosans ${ }^{60,61}$, small viruses ${ }^{62}$ and most types of synthetic or processed polymers ${ }^{63,64,65,66}$. An extensive bibliography may be found in the literature ${ }^{67}$ and online (at http:// www.wyatt.com/bibliography).

Here, we present a standard protocol for running and analyzing a SEC-MALS experiment. Bovine serum albumin (BSA) is presented as an example for separation and characterization of protein monomers and oligomers. The BSA protocol determines certain system constants that serve as a foundation for further SEC-MALS analyses including those of complexes, glycoproteins and surfactant-bound membrane proteins.

We note that SEC-MALS may be performed using standard high-performance liquid chromatography (HPLC) or fast protein liquid chromatography (FPLC) equipment from many vendors. This protocol describes the use of an FPLC system commonly found in labs that produce proteins for research and development (see Table of Materials). Prior to running the protocol, the FPLC system, MALS and dRI 
detectors should have been installed, along with their respective software packages for control, data acquisition and analysis per manufacturers' instructions and any requisite calibration constants or other settings entered into the software. An inline filter should be placed between the pump and injector with a hydrophilic, $0.1 \mu \mathrm{m}$ pore membrane installed.

\section{Protocol}

\section{Preparation of the system}

1. Connect the MALS and dRI detectors downstream of the FPLC's UV detector. Bypass the pH and conductivity detectors since they will add significant interdetector volume between the UV and MALS detectors. Use capillary tubing of $0.25 \mathrm{~mm}$ i.d. from the column to and between the detectors, and $0.75 \mathrm{~mm}$ i.d. capillary tubing on the output of the detectors to waste or fraction collector.

2. Ensure that the necessary signal connections between the FPLC and detectors have been established, including analog output from the UV detector to the MALS analog input, and digital output from the FPLC to the MALS Autoinject, via the FPLC's I/O Box.

3. Install a suitable analytical SEC column covering a fractionation range of at least $20 \mathrm{kDa}$ to $500 \mathrm{kDa}$. Check the product info to determine if the column is suitable for the range of $\mathrm{MW}, \mathrm{pH}$ and other properties of the sample and mobile phase.

\section{Preparation of buffer, $f$ lushing the system overnight and checking cleanliness}

1. Using HPLC-grade reagents, prepare $1 \mathrm{~L}$ of phosphate-buffered saline with $50-100 \mathrm{mM} \mathrm{NaCl}$. Filter the buffer to $0.1 \mu \mathrm{m}$ using a bottle-top polyether sulfone filter or similar. Filter the first $50-100 \mathrm{~mL}$ of buffer to a waste bottle and discard, in order to eliminate particulates from the dry filters, and then filter the remainder to a clean, sterile bottle that has been washed thoroughly with filtered, de-ionized water and capped to prevent dust from entering.

NOTE: Other mobile phase solvents such as a Tris buffer may be used if additional proteins that are preferentially dissolved in those solvents are to be analyzed.

2. Flush overnight at a flow rate of $0.5 \mathrm{~mL} / \mathrm{min}$, or as otherwise recommended by the column manufacturer, to equilibrate the column in the buffer and remove particulates. Use the FPLC's Continuous flow mode and ensure that the flow does not stop until all SEC-MALS runs are complete.

1. Place the dRI flow cell in Purge mode during the overnight flush. Turn the purge off before beginning sample runs.

2. When beginning the flush, gradually ramp the flow rate to prevent "column shedding" effect (or release of particles) caused by a sudden change of pressure in the column.

3. If the system is known to be quite stable and particle-free, and in equilibrium with the desired mobile phase, replace the overnight flush with a shorter, 2-3 $\mathrm{h}$ flush.

3. Check system cleanliness by lightly tapping the tubing downstream of the column to release accumulated particles and observing the signal in the $90^{\circ}$ detector on the front-panel display of the MALS instrument. Verify that the peak-to-peak noise is no more than $50-100 \mu \mathrm{V}$.

4. Perform a 'blank' injection to verify that the injector is clean of particles. A 'blank' is simply the running buffer, prepared in a fresh, sterile vial.

1. If the particle peak is no more than $1 \mathrm{~mL}$ in volume and no more than $5 \mathrm{mV}$ above baseline, then the system is ready for samples. Otherwise, perform additional blank injections until clean, or perform maintenance to clean the injector.

\section{Preparing and loading the sample}

1. Prepare at least $200 \mu \mathrm{L}$ of BSA at $1-2 \mathrm{mg} / \mathrm{mL}$ in the SEC buffer.

NOTE: In order to prevent precipitation, BSA should never be dissolved in pure water.

2. Filter the protein to $0.02 \mu \mathrm{m}$ using a syringe-tip filter.

3. Discard the first few drops of filtrate in order to eliminate particles from the dry filters.

4. Alternatively, centrifuge the sample at $10,000 \times g$ for $15 \mathrm{~min}$ to enable precipitation of non-soluble aggregates and other large particles.

5. Inject $100 \mu \mathrm{L}$ of the BSA solution into the loop.

NOTE: This is the recommended amount of material, and more or less may be injected according to circumstances of the sample such as stability or availability. The quantity of protein required per injection varies inversely with molecular weight - twice as much protein mass is needed if the molecular weight is $33 \mathrm{kDa}$, or half that of BSA.

\section{Preparation of the MALS software}

1. Open New | Experiment from Method in the MALS software menu and select the Online method from the Light Scattering system methods folder. If a DLS detector is present, select the Online method from the Light Scattering | With QELS folder.

2. In the Configuration section, set parameters of the sample and mobile phase.

1. In the Generic Pump view, set the flow rate to that used in the FPLC.

2. In the Generic Pump view, Solvent branch, Name field, select PBS.

3. In the Injector view, Sample branch, enter the Name as BSA, and set $d n / d c=0.185$ (the standard value for unmodified proteins), A2 $=$ 0 , and UV extinction coefficient $=0.667 \mathrm{~mL} /(\mathrm{mg}-\mathrm{cm})$.

NOTE: For other proteins, the $\mathrm{UV}_{280} \mathrm{~nm}$ extinction coefficient may be found in the literature or calculated from its sequence using various public-domain software tools.

3. In the Procedures section, Basic Collection view, select the checkbox Trigger on Autoinject and set the duration of the run to 70 min so that data are collected for the entire elution until the total permeation volume of the SEC column is reached. 
NOTE: The necessary amount of time may vary with column and flow rate $-35 \mathrm{~min}$ of collection are required for a standard $7.8 \mathrm{~mm} \times 300 \mathrm{~mm}$ HPLC-SEC column at $0.5 \mathrm{~mL} / \mathrm{min}$.

4. Start the experiment in the MALS software by clicking on the Run button. It will start reading the data after receiving the pulse signal from the FPLC instrument via the MALS detector.

5. Zero the dRI signal by clicking the Autozero button on the instrument's front panel.

\section{Preparation of the FPLC software}

1. Insert the name of protein and the run in the FPLC software, in Manual | Execute manual instructions | Set mark.

2. Switch the injection valve from Manual load to Inject under Flow path | Injection valve.

3. Include a pulse signal by inserting a $0.5 \mathrm{~s}$ pulse under I/O box | Pulse digital out. This will trigger data collection in the MALS software.

\section{Inject the sample into the loop. Click Execute in the FPLC software to start the experiment run.}

\section{Analysis of SEC-MALS BSA data}

1. Perform analysis, step by step, under the Procedures section in MALS software.

1. Verify that peaks appear, at approximately the same elution volume in UV, MALS and RI, by checking the Basic Collection view.

2. In the Baseline view, define baseline for all signals (all LS detectors, UV and dRI). The baselines should be defined to indicate the level of pure solvent, preferable stretching from one side of the sample peaks to the other.

3. In the Peaks view, define the peaks to be analyzed by clicking and dragging the mouse. Select the central $50 \%$ of each peak. First select the monomer peak ('Peak 1') and then the dimer peak ('Peak 2'). Verify correct values of $d n / d c=0.185$ and UV $280 \mathrm{~nm}$ extinction coefficient $=0.667$ for BSA under each peak

2. Perform peak alignment, band-broadening correction and normalization procedures

NOTE: Normally the SEC-MALS method is periodically calibrated for peak alignment, band broadening and normalization of the angular detectors to the $90^{\circ}$ detector using a monodisperse protein with radius of gyration $R_{\mathrm{g}}<10 \mathrm{~nm}$ such as BSA monomer. In this example, BSA serves both as the calibration molecule and is itself the subject of MW analysis.

1. In the Procedures | Alignment view, select the central region of the peaks by clicking and dragging the mouse, click Align Signals and then OK.

2. In the Procedures | Band Broadening view, choose the central $50 \%$ of the monomer peak. Make sure the RI detector is specified as the Reference Instrument, then click Perform Fit and Apply to match the UV and LS signals to the RI signal.

1. Zoom in to the peaks to verify that they overlap very closely within the central $50-70 \%$, then click OK.

2. If the overlap is not perfect, (it may be necessary to) perform the fit and "Apply" one or two more times until the overlap is excellent.

3. In the Procedures | Normalization view, select Peak 1, enter $3.0 \mathrm{~nm}$ as the $R_{\mathrm{g}}$ value, click Normalize then OK.

4. In the Procedures | Molar Mass and Rg from MALS view, review the data to determine which, if any, detection angles should be deselected from the analysis due to excessive noise. Typically, these will be the lower angles into which dust particles scatter a relatively high intensity. Select individual slices within the peaks from the graph on the right and view the angular dependence of the inverse reduced Rayleigh ratio in the graph on the left. If the lowest (and sometimes the highest) angles consistently deviate greatly from the fit, then deselect them from the list at the bottom of the view.

3. View the graph of the results in the EASI Graph view. Select Molar Mass from the Display drop-down at the top of the window. Use Ctrl + click and drag to zoom in on the peak region.

4. View the final tabulated weight-average molar mass results for the monomer and dimer peaks in the Results | Report (summary) view under Peak Results | Molar mass moments $(\mathbf{g} / \mathbf{m o l})$ | Mw. Purity is reported under Peak Results | Mass fraction(\%).

NOTE: Other molar mass moments are also shown; these are usually relevant to heterogeneous polymers, but not to proteins with discrete sizes. Many other results produced by the software may be included in the report such as percent mass recovery (the fraction of protein that eluted via the concentration detector relative to the amount injected), peak statistics, polydispersity, $R_{\mathrm{g}}$ and $R_{\mathrm{h}}$ moments, etc. When provided, the measures of uncertainty reflect the precision of the value cited based on the noise within the measurement series and should not be considered to represent the accuracy. The true accuracy of the reported values depends on various factors such as the accuracy of the provided $d n / d c$ and extinction coefficient values, instrument calibration, etc.

5. From the File menu, select Save as Method and save the analyzed BSA data as a standard method for future measurements of all types of proteins. The normalization and band-broadening parameters determined for BSA will be carried over in the analysis.

\section{Representative Results}

Figure 1a,b show that three oligomeric forms of BSA: monomer, dimer and trimer, were well-separated on the $200 \AA$ pore column with baseline resolution of monomer and dimer, while Figure 2a shows that separation on a $75 \AA$ pore column did not achieve good monomer-dimer resolution. The latter example was included to illustrate a "poor" result; these differences in separation for the two columns may, in fact, be expected according to the manufacturer's stated separation ranges. The trimer is not fully separated from the dimer and higher oligomers are not well separated from the trimer and each other. Figure $\mathbf{2 b}$ is an example of noisy light scattering signal with particles present throughout the chromatogram, which precludes accurate MW determination. 
Henceforth we focus on Figure 1b. The monomer, which eluted at $13.8 \mathrm{~mL}$, exhibits a weight-average molar mass $M_{\mathrm{w}}$ of $64.1 \pm 0.4 \mathrm{kDa}$ determined by MALS and hydrodynamic radius $R_{\mathrm{h}}$ of $3.54 \pm 0.01 \mathrm{~nm}$. These results are in agreement with the sequence mass and known hydrodynamic radius of BSA, $66.4 \mathrm{kDa}$ and $3.5 \mathrm{~nm}$ respectively ${ }^{68}$, to within the usual accuracy of SEC-MALS, $5 \%{ }^{3,69}$. The dimer, which eluted at $12 \mathrm{~mL}$, exhibited a $M_{\mathrm{w}}$ value of $127 \pm 1 \mathrm{kDa}$ determined by MALS-as expected, twice that of the monomer to within experimental precisionand $R_{\mathrm{h}}$ of $5.68 \pm 0.06 \mathrm{~nm}$. The trimer peak was also observed at $11 \mathrm{~mL}$ with $M_{\mathrm{w}}$ of $194 \pm 9 \mathrm{kDa}$ determined by MALS, three times that of the monomer to within experimental precision, as expected. $R_{\mathrm{h}}$ of the trimer could not be determined due to low intensity of the DLS signal.

The molar mass points calculated across the monomer peak are uniform to within $2-5 \%$, indicating homogeneity. It is not unusual to find a trailing shoulder with molar mass in the range of $38-50 \mathrm{kDa}$, corresponding to BSA fragments ${ }^{70}$. The molar mass points across the dimeric and trimeric peaks are not uniform, indicative of heterogeneity. The dimer peak is somewhat heterogeneous due to traces of trimer that bleed into the dimer peak, and the trimer peak is heterogeneous due to co-elution of poorly-resolved higher oligomers.

The signal-to-noise level of the monomer peak is quite acceptable in all three signals, over 100:1, as is the dimer peak with signal-to-noise of 40:1. Chromatogram regions beyond the protein peaks are flat, with the exceptions of a peak due to particulates near the total exclusion (void) volume in the LS trace and a (positive) salt peak and a (negative) dissolved air peak in the dRI trace, near the total permeation volume. These are pointed out in Figure 1a.

Level of purity can also be calculated in a SEC-MALS experiment: mass fraction of the monomeric peak in the report represents the percent of purity of the monomeric form. For BSA monomer, the calculated purity is $88 \%$.
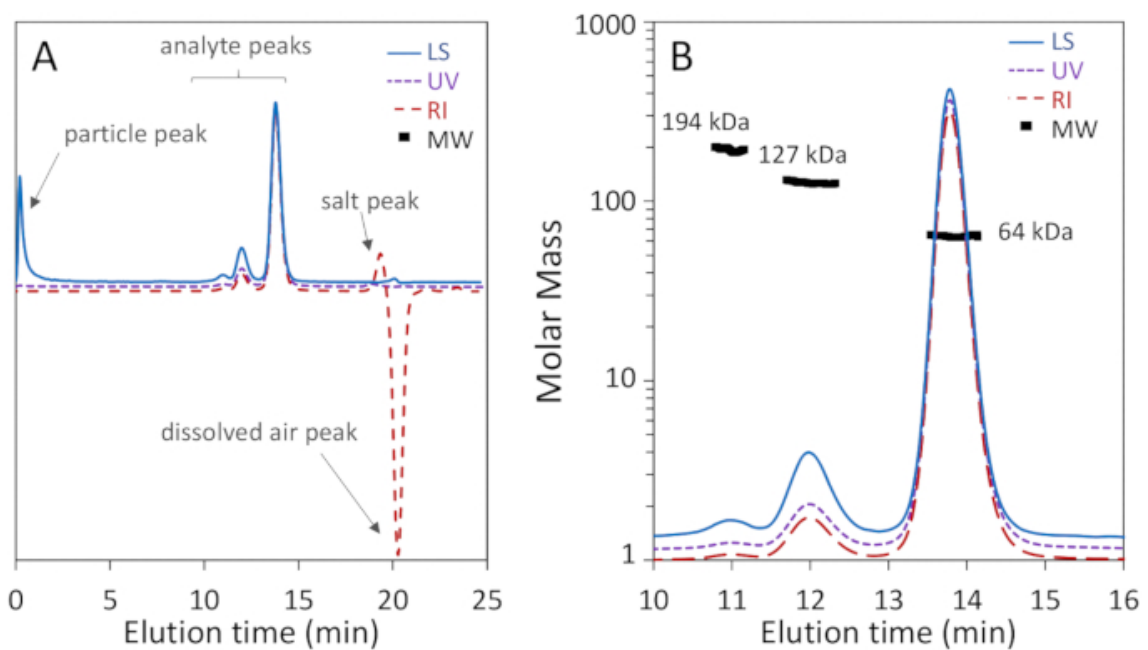

Figure 1: SEC-MALS analysis of bovine serum albumin (BSA) using a 200 A pore size-exclusion column. Chromatogram traces are normalized to the monomer peak and offset for clarity. (A) Common artifacts that may be ignored are pointed out, including a particle peak near the beginning of the light scattering signal as well as salt and dissolved air peaks near the total permeation volume in the refractive index signal. (B) The chromatogram exhibits excellent monomer-dimer-trimer separation and the light scattering signal exhibits high signal-to-noise. The monomer and dimer MW values exhibit high homogeneity. Please click here to view a larger version of this figure. 

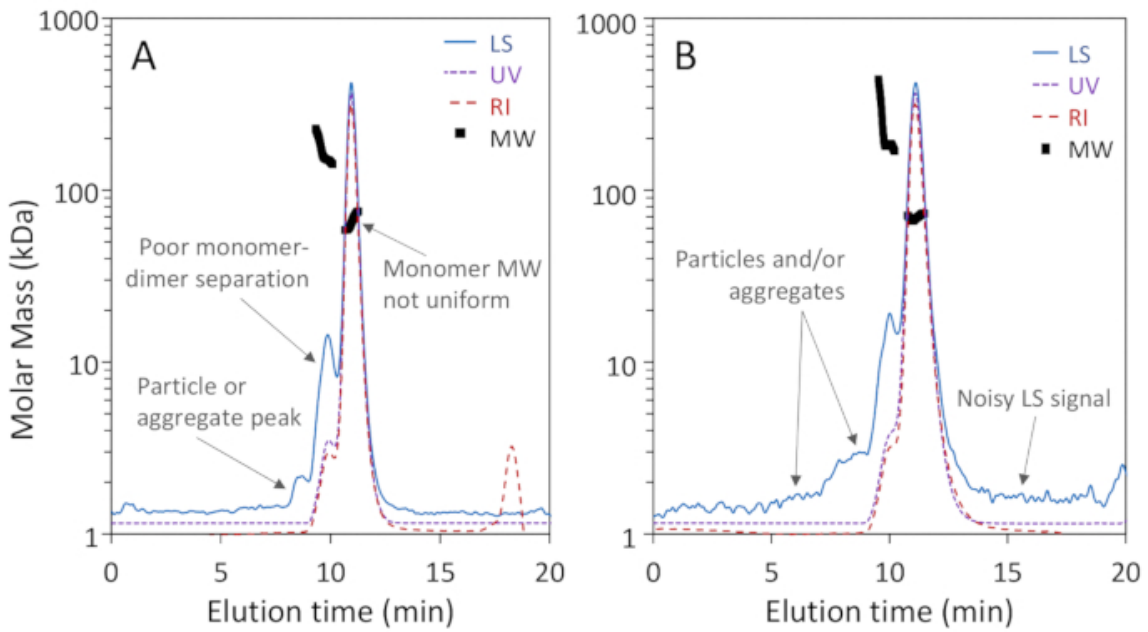

Figure 2: Examples of low-quality SEC-MALS analyses. Chromatogram traces are normalized to the monomer peak and offset for clarity. (A) Inadequate separation on a $75 \AA$ pore size exclusion column; a particle peak between 8 - 9 min is not well separated from the proteins. (B) Inadequate signal-to-noise ratio and extensive particles adjacent to the proteins are apparent in the light scattering (LS) signal. Please click here to view a larger version of this figure.

\section{Discussion}

The SEC-MALS experiment has provided good separation of monomer, dimer and trimer, and quantitative results for the molar masses and hydrodynamic sizes of each peak. This in turn clearly identifies and characterizes each species present, as well as quantifying purity. Usually the results obtained are accurate to within $5 \%$, and precise and repeatable to within $1-2 \% 3,69$. This level of precision and repeatability makes it possible to confidently distinguish between species that may be close in MW, as long as they are separated by SEC (may partially overlap within the same peak). The benefits for protein quality control and fundamental biophysical characterization are apparent.

Verification of the absence of particulates is quite important for sensitive, repeatable SEC-MALS measurements. Particle peaks generally appear as large MALS signals unaccompanied by comparable UV or RI signals. The mobile phase and sample should be prepared carefully to eliminate such particles. Use of HPLC-grade reagents or better, filtration of mobile phase and dilution buffer to $0.1-0.2 \mu \mathrm{m}$ (pre-washing the filter to eliminate particles that are always present on dry membranes), maintenance of extra-clean mobile phase bottles and other glassware for SEC-MALS and extended column equilibration under flow (to remove particulates and aggregates that may have accumulated when flow was stopped or a column not in use) are all recommended. The sample should be filtered to the smallest pore size that does not remove the material of interest, usually no larger than $0.1 \mu \mathrm{m}$ and, if possible, $0.02 \mu \mathrm{m}$. If the filter quickly clogs, the sample may be centrifuged, filtered in stages of descending pore size, and/or re-purified. When systems are consistently maintained with high-quality, fresh and filtered mobile phase, column shocks are prevented, samples are clean and do not adhere to the column, the measurements will not exhibit the aforementioned particulate noise and will provide high-quality data.

MALS is agnostic to typical SEC buffers for proteins; many other buffer systems besides PBS can be used to optimize separation and stability, including a variety of excipients ${ }^{71}$. MALS is also agnostic to the specific column, which should be selected for optimal separation and recovery. The primary concerns for excipients regarding analysis are significant changes in refractive index, leading to modification of the specific refractive index increment $d n / d c$, and excipients that absorb $280 \mathrm{~nm}$ when UV analysis is needed. For example, arginine is a common aggregation-reducing excipient that can dramatically affect the $d n / d c$ of a typical protein, even bringing it into the negative regime (a protein with negative $d n / d c$ can still be analyzed by SEC-MALS if $d n / d c$ is determined empirically, but if $d n / d c=0$ the intensity of light scattered by the protein will be null and MW analysis will be impossible). The topic of $d n / d c$ values for proteins is discussed extensively by Zhao et al. ${ }^{35}$ where it is shown that for standard aqueous buffers, the vast majority of unmodified proteins fall within $2-3 \%$ of the standard value $(0.185$ or $0.186 \mathrm{~mL} / \mathrm{g}$ at $=660$ $\mathrm{nm}$ ), though proteins below $\sim 10 \mathrm{kDa}$ are more variable, and there are a few species that may go as high as $0.21 \mathrm{~mL} / \mathrm{g}$.

The MW profiles across the BSA monomer and dimer peaks in the data presented were both quite homogeneous to within $2 \%$ or less, indicating monodisperse species. Non-uniform MW values across a peak may arise from heterogeneity or improper analysis. In particular, a BSA MW profile that is concave ('smile') or convex ('grimace') could result from not correctly applying the band-broadening correction. For other proteins, a convex profile could also arise from dynamic equilibrium between monomers and oligomers, where the ratio of monomer to oligomer-and hence the apparent MW value-depends on peak concentration (BSA does not exhibit this behavior and is often used as a control sample to verify correct band broadening parameters). A MW profile that varies from the leading to the trailing edge and does not change with sample concentration is typical of a distribution of molecular weight species that are partially resolved by SEC. Dynamic equilibrium is readily distinguished from the other sources of apparently inhomogeneous distributions by injecting different total quantities of protein-the distribution will vary with dynamic equilibrium but not with a fixed distribution or incorrect band-broadening correction.

Given the constraints described above, SEC-MALS is not suitable for various tasks. It is not suitable for analysis of crude samples; the samples should be well-purified by standard affinity and polishing methods. It does not have sufficient accuracy or resolving power to identify mutants and variants of a protein or mAb with same or very close mass, and cannot be used with analytes that do not elute from or separate on a SEC column, though recently it has been shown that ion-exchange or reverse-phase chromatography can be combined with MALS to separate and characterize species that are not resolved by SEC ${ }^{72,73,74}$. Where protein quantities are severely constrained, SEC-MALS may not be feasible 
since it typically requires $10-200 \mathrm{\mu g}^{3}$ and even more may be required by an FPLC system with tubing inner diameter greater than 0.25 mm; however, smaller quantities may be analyzed by UHPLC-SEC-MALS. Highly unstable proteins that aggregate upon introduction to the mobile phase are not suitable for SEC-MALS analysis, though buffer optimization using off-line dynamic light scattering may overcome this problem ${ }^{75}$.

Despite the additional effort that SEC-MALS entails, it is invaluable for protein research and is used extensively by the academic and biopharmaceutical communities. In addition to characterization of monomers, oligomers and aggregates as described in the protocol above, SEC-MALS can characterize modified proteins such as glycoproteins (determining the MW of the protein and glycan components individually), surfactant- or lipid-solubilized membrane proteins (determining the MW of the protein and solubilizer components individually), protein assemblies such as virus-like particles, protein-protein and protein-nucleic acid complexes, polysaccharides, protein-polysaccharide conjugates, peptides and many other biomacromolecules.

\section{Disclosures}

DS is an employee of Wyatt Technology Corporation, whose MALS products are utilized in this protocol. AT is an employee of Danyel Biotech, a distributor of Wyatt MALS and AKTA FPLC instruments.

\section{Acknowledgments}

We thank Dr. Tsafi Danieli (Wolfson Centre for Applied Structural Biology, Hebrew University) for advice and collaborations. We also thank Daniel Biotech Ltd. (Rehovot, Israel) for the assistance and establishment of the analytical FPLC-MALS system utilized in this study.

\section{References}

1. Acton, T.B. et al. Robotic cloning and Protein Production Platform of the Northeast Structural Genomics Consortium. Methods in Enzymology. 394, 210-243 (2005).

2. Acton, T.B. et al. Preparation of protein samples for NMR structure, function, and small-molecule screening studies. Methods in Enzymology. 493, 21-60 (2011).

3. Folta-Stogniew, E., Williams, K.R. Determination of molecular masses of proteins in solution: Implementation of an HPLC size exclusion chromatography and laser light scattering service in a core laboratory. Journal of Biomolecular Technology. 10 (2), 51-63, at <https:// www.ncbi.nlm.nih.gov/pubmed/19499008> (1999).

4. Kendrick, B.S., Kerwin, B.A., Chang, B.S., Philo, J.S. Online size-exclusion high-performance liquid chromatography light scattering and differential refractometry methods to determine degree of polymer conjugation to proteins and protein-protein or protein-ligand association states. Analytical Biochemistry. 299 (2), 136-146 (2001).

5. Hughes, C.S., Longo, E., Phillips-Jones, M.K., Hussain, R. Quality control and biophysical characterisation data of. Data Brief. 14, 41-47 (2017).

6. Muthurajan, U. et al. In Vitro Chromatin Assembly: Strategies and Quality Control. Methods in Enzymology. 573, 3-41 (2016).

7. P4EU | Protein Quality Standard PQS. at <https://p4eu.org/protein-quality-standard-pqs> (2019).

8. Arbre Mobieu | Guidelines on Protein Quality Control. at <https://arbre-mobieu.eu/guidelines-on-protein-quality-control/> (2019).

9. Bowman, A. et al. The histone chaperones Vps75 and Nap1 form ring-like, tetrameric structures in solution. Nucleic Acids Research. 42 (9), 6038-6051 (2014).

10. Bowman, G.R. et al. Oligomerization and higher-order assembly contribute to sub-cellular localization of a bacterial scaffold. Molecular Microbiology. 90 (4), 776-795 (2013).

11. Folta-Stogniew, E. Oligomeric states of proteins determined by size-exclusion chromatography coupled with light scattering, absorbance, and refractive index detectors. Methods in Molecular Biology. 328, 97-112 (2006).

12. Vieux, E.F., Wohlever, M.L., Chen, J.Z., Sauer, R.T., Baker, T.A. Distinct quaternary structures of the AAA+ Lon protease control substrate degradation. Proceedings of the National Academy of Sciences of the United States of America. 110 (22), E2002-8 (2013).

13. Group, N.S. use of SEC-MALS in crystallization QC. 2018 (December 9), at <http://www.nesg.org/documents/ protein_aggregation_screening.pdf> (2018).

14. Ahrer, K., Buchacher, A., Iberer, G., Josic, D., Jungbauer, A. Analysis of aggregates of human immunoglobulin $G$ using size-exclusion chromatography, static and dynamic light scattering. Journal of Chromatography A. 1009 (1-2), 89-96, at <https://www.ncbi.nlm.nih.gov/ pubmed/13677648> (2003).

15. Narhi, L.O., Schmit, J., Bechtold-Peters, K., Sharma, D. Classification of protein aggregates. Journal of Pharmaceutical Sciences. 101 (2), 493-8 (2012).

16. Spiess, C. et al. Bispecific antibodies with natural architecture produced by co-culture of bacteria expressing two distinct half-antibodies. Nature Biotechnology. 31 (8), 753-758 (2013).

17. Philo, J.S. A critical review of methods for size characterization of non-particulate protein aggregates. Current Pharmaceutical Biotechnology. 10 (4), 359-372, at <https://www.ncbi.nlm.nih.gov/pubmed/19519411> (2009).

18. Stellwagen, E. Gel filtration. Methods in Enzymology. 463, 373-385 (2009).

19. Burgess, R.R. A brief practical review of size exclusion chromatography: Rules of thumb, limitations, and troubleshooting. Protein Expression and Purification. 150, 81-85 (2018).

20. Striegel, A.M., Yau, W.W., Kirkland, J.J., Bly, D.D. Modern Size-Exclusion Liquid Chromatography. John Wiley \& Sons, Inc. Hoboken, NJ, USA. (2009).

21. Healthcare, G. Size Exclusion Chromatography: Principles and Methods. at <https://cdn.gelifesciences.com/dmm3bwsv3/AssetStream.aspx? mediaformatid=10061\&destinationid=10016\&assetid=11639> (2014).

22. Uliyanchenko, E. Size-exclusion chromatography-from high-performance to ultra-performance. Analytical and Bioanalytical Chemistry. 406 (25), 6087-6094 (2014). 
23. Dunker, A.K., Silman, I., Uversky, V.N., Sussman, J.L. Function and structure of inherently disordered proteins. Current Opinion in Structural Biology. 18 (6), 756-764 (2008).

24. Hsiao, H.H., Nath, A., Lin, C.Y., Folta-Stogniew, E.J., Rhoades, E., Braddock, D.T. Quantitative characterization of the interactions among cmyc transcriptional regulators FUSE, FBP, and FIR. Biochemistry. 49 (22), 4620-4634 (2010).

25. Hayashi, Y., Takagi, T., Maezawa, S., Matsui, H. Molecular weights of alpha beta-protomeric and oligomeric units of soluble (Na+, $\mathrm{K}+$ )ATPase determined by low-angle laser light scattering after high-performance gel chromatography. Biochimica et Biophysica Acta. 748 (2), 153-167 (1983).

26. Folta-Stogniew, E.J. Macromolecular Interactions: Light Scattering. Encyclopedia of Life Sciences. (2009).

27. Hong, P., Koza, S., Bouvier, E.S.P. Size-Exclusion Chromatography for the Analysis of Protein Biotherapeutics and their Aggregates. Journal of Liquid Chromatography and Related Technology. 35 (20), 2923-2950 (2012).

28. Chakrabarti, A. Separation of Monoclonal Antibodies by Analytical Size Exclusion Chromatography. Antibody Engineering. (2018).

29. Wyatt, P.J. Light scattering and the absolute characterization of macromolecules. Analytica Chimica Acta. 272 (1993).

30. Takagi, T. Application of low-angle laser light scattering detection in the field of biochemistry: review of recent progress. Journal of Chromatography A. 506, 51-63 (1990).

31. Wen, J., Arakawa, T., Philo, J.S. Size-exclusion chromatography with on-line light-scattering, absorbance, and refractive index detectors for studying proteins and their interactions. Analytical Biochemistry. 240 (2), 155-166 (1996).

32. Mogridge, J. Using light scattering to determine the stoichiometry of protein complexes. Methods in Molecular Biology. 1278, 233-238 (2015).

33. Larkin, M., Wyatt, P.J. Light-Scattering Techniques and their Application to Formulation and Aggregation Concerns. Formulation and Process Development Strategies for Manufacturing Biopharmaceuticals. (2010).

34. Wolfender, J.-L. HPLC in Natural Product Analysis: The Detection Issue. Planta Medica. 75 (07), 719-734 (2009).

35. Zhao, H., Brown, P.H., Schuck, P. On the distribution of protein refractive index increments. Biophysical Journal. 100 (2011).

36. Serebryany, E., Folta-Stogniew, E., Liu, J., Yan, E.C. Homodimerization enhances both sensitivity and dynamic range of the ligand-binding domain of type 1 metabotropic glutamate receptor. FEBS Letters. 590 (23), 4308-4317 (2016).

37. Arakawa, T., Wen, J. Size-exclusion chromatography with on-line light scattering. Current Protocols in Protein Science. Chapter 20, Unit 20.6 (2001).

38. Müller, R. et al. High-resolution structures of the IgM Fc domains reveal principles of its hexamer formation. Proceedings of the National Academy of Sciences. 110 (25), 10183-10188 (2013).

39. Slotboom, D.J., Duurkens, R.H., Olieman, K., Erkens, G.B. Static light scattering to characterize membrane proteins in detergent solution. Methods. 46 (2), 73-82 (2008).

40. Miercke, L.J., Robbins, R.A., Stroud, R.M. Tetra detector analysis of membrane proteins. Current Protocols in Protein Science. 77, 29.10.1-30 (2014).

41. Gimpl, K., Klement, J., Keller, S. Characterising protein/detergent complexes by triple-detection size-exclusion chromatography. Biological Procedures Online. 18 (1), 4 (2016).

42. Korepanova, A., Matayoshi, E.D. HPLC-SEC characterization of membrane protein-detergent complexes. Current Protocols in Protein Science. Chapter 29, Unit 29.5.1-12 (2012).

43. Roy, A., Breyton, C., Ebel, C. Analytical Ultracentrifugation and Size-Exclusion Chromatography Coupled with Light Scattering for Characterization of Membrane Proteins in Solution. Membrane Proteins Production for Structural Analysis. (2014).

44. Hastie, K.M. et al. Crystal structure of the prefusion surface glycoprotein of the prototypic arenavirus LCMV. Nature Structural \& Molecular Biology. 23 (6), 513-521 (2016).

45. Pallesen, J. et al. Structures of Ebola virus GP and sGP in complex with therapeutic antibodies. Nature Microbiology. 1 (9), 16128 (2016).

46. Micoli, F., Adamo, R., Costantino, P. Protein Carriers for Glycoconjugate Vaccines: History, Selection Criteria, Characterization and New Trends. Molecules. 23 (6) (2018).

47. Li, J. et al. Characterizing the Size and Composition of Saposin A Lipoprotein Picodiscs. Analytical Chemistry. 88 (19), $9524-9531$ (2016).

48. Crichlow, G. V et al. Dimerization of FIR upon FUSE DNA binding suggests a mechanism of c-myc inhibition. EMBO Journal. 27 (1), $277-289$ (2008).

49. Kapoor, N., Gupta, R., Menon, S.T., Folta-Stogniew, E., Raleigh, D.P., Sakmar, T.P. Nucleobindin 1 is a calcium-regulated guanine nucleotide dissociation inhibitor of G\{alpha\}i1. Journal of Biological Chemistry. 285 (41), 31647-31660 (2010).

50. Pirruccello, M., Swan, L.E., Folta-Stogniew, E., De Camilli, P. Recognition of the F\&H motif by the Lowe syndrome protein OCRL. Nature Structural \& Molecular Biology. 18 (7), 789-795 (2011).

51. Lockyer, K., Gao, F., Derrick, J.P., Bolgiano, B. Structural correlates of carrier protein recognition in tetanus toxoid-conjugated bacterial polysaccharide vaccines. Vaccine. 33 (11), 1345-1352 (2015).

52. Steinbach, T., Wurm, F.R. Degradable Polyphosphoester-Protein Conjugates: "PPEylation" of Proteins. Biomacromolecules. 17 (10), 3338-3346 (2016).

53. Das, S., Stivison, E., Folta-Stogniew, E., Oliver, D. Reexamination of the role of the amino terminus of SecA in promoting its dimerization and functional state. Journal of Bacteriology. 190 (21), 7302-7307 (2008).

54. Reshetnyak, A. V et al. The strength and cooperativity of KIT ectodomain contacts determine normal ligand-dependent stimulation or oncogenic activation in cancer. Molecular Cell. 57 (1), 191-201 (2015).

55. Zambelli, B. et al. UreG, a Chaperone in the Urease Assembly Process, Is an Intrinsically Unstructured GTPase That Specifically Binds Zn2+. Journal of Biological Chemistry. 280 (6), 4684-4695 (2005).

56. Ren, X. et al. Hybrid Structural Model of the Complete Human ESCRT-0 Complex. Structure. 17 (3), 406-416, (2009).

57. Moriarty, D.F., Fiorillo, C., Miller, C., Colón, W. A truncated peptide model of the mutant P61A FIS forms a stable dimer. Biochimica et Biophysica Acta (BBA) - Proteins and Proteomics. 1774 (1), 78-85, (2007).

58. la Garza, C.E., Miranda-Hernández, M.P., Acosta-Flores, L., Pérez, N.O., Flores-Ortiz, L.F., Medina-Rivero, E. Analysis of therapeutic proteins and peptides using multiangle light scattering coupled to ultra high performance liquid chromatography. Journal of Separation Science. 38 (9), 1537-1543 (2015).

59. Beirne, J., Truchan, H., Rao, L. Development and qualification of a size exclusion chromatography coupled with multiangle light scattering method for molecular weight determination of unfractionated heparin. Analytical and Bioanalytical Chemistry. 399 (2), 717-725 (2011).

60. Wang, W. et al. A new green technology for direct production of low molecular weight chitosan. Carbohydrate Polymers. 74 (1), 127-132, (2018). 
61. Kaderli, S. et al. A novel biocompatible hyaluronic acid-chitosan hybrid hydrogel for osteoarthrosis therapy. International Journal of Pharmaceutics. 483 (1-2), 158-168 (2015).

62. Porterfield, J.Z., Zlotnick, A. A Simple and General Method for Determining the Protein and Nucleic Acid Content of Viruses by UV Absorbance. Virology. 407 (2), 281-288 (2010).

63. Podzimek, S. The use of GPC coupled with a multiangle laser light scattering photometer for the characterization of polymers. On the determination of molecular weight, size and branching. Journal of Applied Polymer Science. 54 (1), 91-103 (1994).

64. Podzimek, S., Vlcek, T., Johann, C. Characterization of branched polymers by size exclusion chromatography coupled with multiangle light scattering detector. I. Size exclusion chromatography elution behavior of branched polymers. Journal of Applied Polymer Science. 81 (7), 1588-1594 (2001).

65. Podzimek, S. Importance of Multi-Angle Light Scattering in Polyolefin Characterization. Macromolecular Symposia. 330 (1), $81-91$ (2013).

66. Tarazona, M.P., Saiz, E. Combination of SEC/MALS experimental procedures and theoretical analysis for studying the solution properties of macromolecules. Journal of Biochemical and Biophysical Methods. 56 (1), 95-116, (2013).

67. Minton, A.P. Recent applications of light scattering measurement in the biological and biopharmaceutical sciences. Analytical Biochemistry. 501, 4-22 (2016)

68. Hirayama, K., Akashi, S., Furuya, M., Fukuhara, K. Rapid confirmation and revision of the primary structure of bovine serum albumin by ESIMS and frit-FAB LC/MS. Biochemical and Biophysical Research Communications. 173 (2), 639-646 (1990).

69. Zhu, H., Ownby, D.W., Riggs, C.K., Nolasco, N.J., Stoops, J.K., Riggs, A.F. Assembly of the gigantic hemoglobin of the earthworm Lumbricus terrestris. Roles of subunit equilibria, non-globin linker chains, and valence of the heme iron. The Journal of biological chemistry. 271 (47), 30007-21 (1996).

70. Peters, T., Feldhoff, R.C. Fragments of bovine serum albumin produced by limited proteolysis. Isolation and characterization of tryptic fragments. Biochemistry. 14 (15), 3384-91 (1975).

71. Lebendiker, M., Danieli, T. Production of prone-to-aggregate proteins. FEBS Letters. 588 (2), 236-246 (2014).

72. Amartely, H., Avraham, O., Friedler, A., Livnah, O., Lebendiker, M. Coupling Multi Angle Light Scattering to lon Exchange chromatography (IEX-MALS) for protein characterization. Scientific Reports. 8 (1), 6907 (2018).

73. Astafieva, I. V., Eberlein, G.A., John Wang, Y. Absolute on-line molecular mass analysis of basic fibroblast growth factor and its multimers by reversed-phase liquid chromatography with multi-angle laser light scattering detection. Journal of Chromatography A. 740 (2), 215-229 (1996).

74. Onsberg, M., Øgendal, L.H., Jensen, M.L., Howells, L.B., Andersen, B., Bjerrum, M.J. Light scattering coupled with reversed phase chromatography to study protein self-association under separating conditions. Journal of Chromatography B. 938, 60-64 (2013).

75. Kim, Y. et al. High-throughput protein purification and quality assessment for crystallization. Methods. 55 (1), 12-28 (2011). 